\title{
Disease characteristics and medication use in a multicenter cohort of children with juvenile idiopathic arthritis (JIA): preliminary analyses from the CARRAnet registry
}

\author{
Sarah Ringold ${ }^{5 *}$, Timothy Beukelman ${ }^{7}$, Esi M Morgan DeWitt ${ }^{2}$, Marc Natter ${ }^{1}$, Peter A Nigrovic ${ }^{6}$, Yukiko Kimura ${ }^{3}$, \\ CARRAnet Investigators ${ }^{4}$
}

From 2011 Pediatric Rheumatology Symposium sponsored by the American College of Rheumatology Miami, FL, USA. 2-5 June 2011

\section{Purpose}

The CARRAnet Registry, a multicenter registry of children with rheumatic diseases in the U.S. organized by the Childhood Arthritis Rheumatology and Research Alliance (CARRA), began enrollment in May 2010. Our aims were to describe the characteristics of children with JIA enrolled into the registry to date and to identify characteristics associated with the use of biologic disease-modifying anti-rheumatic drugs (DMARDs).

\section{Methods}

Data were extracted for all children with JIA who were enrolled up to December 28, 2010. Children who had ever received biologic DMARDs were compared to children who had not using relative risks (RR) and unpaired t-tests.

\section{Results}

1072 children with JIA were enrolled during the first 7 months by 26 centers. The categorical characteristics of the cohort are shown in Table 1 and the continuous characteristics of the cohort are shown in Table 2. 77\% had received at least one non-biologic DMARD at enrollment, most commonly methotrexate. $69 \%$ had received corticosteroids during their disease course, most frequently intra-articular (49\%) and daily oral (36\%). $45 \%$ of the cohort received one or more biologic DMARD during their disease course. The proportion of patients who received specific biologic agents is shown in Table 3 .
Children receiving biologic DMARDs were older at enrollment (mean age 13 years versus 10 years; $p<0.001$ ) and had a longer disease duration (mean 6 years versus 4 years; $\mathrm{p}<0.001$ ). Children with imaging evidence of joint damage (RR 1.6; 95\% CI: 1.3-1.9), positive RF (RR: 1.36; 95\% CI 1.1 - 1.6), or positive anti-CCP (RR: 1.35; 1.1-1.7) were more likely to have received a biologic DMARD. Children with oligoarthritis were less likely to have received a biologic DMARD than other categories.

\section{Conclusion}

The majority of patients with JIA enrolled into the CARRAnet registry has relatively low disease activity, minimal disability, and have received at least one DMARD. Positive anti-CCP or RF, joint damage on imaging, older age at enrollment and longer disease duration were associated with biologic DMARD use. Limitations include the underrepresentation of non-English speaking families and enrollment bias. Continued enrollment into this cohort will support future analyses with increased sample sizes and the potential for longitudinal data analysis.

\section{Disclosure}

Sarah Ringold: None; Timothy Beukelman: None; Esi M. Morgan DeWitt: None; Marc Natter: None; Peter A. Nigrovic: None; Yukiko Kimura: None; CARRAnet Investigators: None.

${ }^{5}$ Seattle Children's Hospital, Seattle, WA, USA

Full list of author information is available at the end of the article

C 2012 Ringold et al; licensee BioMed Central Ltd. This is an Open Access article distributed under the terms of the Creative Commons 
Table 1

\begin{tabular}{ll}
\hline Characteristuc & N (\%) \\
\hline JA Category & $87(8)$ \\
Systemic & $334(31)$ \\
Polyarticular (RF-) & $266(25)$ \\
Polyarticular (RF+) & $91(9)$ \\
Extended Oligoarticular & $56(5)$ \\
Psoriatic & $110(10)$ \\
Enthesitis-Related & $37(3)$ \\
Undifferentiated & $14(1)$ \\
Other or unknown & $783(73)$ \\
Female & \\
Race & $965(89)$ \\
White & $62(6)$ \\
Black or African American & $29(3)$ \\
Asian & $54(5)$ \\
Other & \\
Ethnicity & $101(9)$ \\
Hispanic or Latino & $265(25)$ \\
\hline Positic Serology &
\end{tabular}

Table 2 (Continued)

\begin{tabular}{lll}
\hline $\begin{array}{l}\text { Physician global assessment of disease } \\
\text { activity }\end{array}$ & $2(1)$ & $0-9$ \\
$\begin{array}{l}\text { Parent/patient assessment of disease acticity } \\
\text { Parent/patient assessment of overall well- }\end{array}$ & $2(2)$ & $0-10$ \\
$\begin{array}{l}\text { being } \\
\text { Patrent/patient asessment of pain }\end{array}$ & $3(2)$ & $0-9$ \\
CHAQ score & $0.35(0.125)$ & $0-3$ \\
\hline
\end{tabular}

Table 3

\begin{tabular}{lll}
\hline Biologic medication & Current use & Prior use \\
\hline & $\mathrm{N}(\%$ of biologic users) & $\mathrm{N}(\%$ of biologic users) \\
\hline TNF-alpha inhibitirs & & \\
Adalimumab & $78(7)$ & $70(6)$ \\
Cartolizumab & $4(<1)$ & 0 \\
Etanercept & $214(20)$ & $183(17)$ \\
Gollimumab & $5(<1)$ & $4(<1)$ \\
Inflximab & $46(4)$ & $46(4)$ \\
IL-1 Inhibitors & & \\
Ankira & $14(1)$ & $22(2)$ \\
Rilonacept & $2(<1)$ & $2(<1)$ \\
Other biologic agents & & \\
Abatacept & $24(2)$ & $8(<1)$ \\
Rituximab & $2(<1)$ & $8(<1)$ \\
Tocilizumab & $6(<1)$ & $0(<1)$ \\
\hline
\end{tabular}

\section{Author details}

${ }^{1}$ Children's Hospital Boston, Boston, MA, USA. ${ }^{2}$ Cincinnati Children'sHospital, Cincinnati, OH, USA. ${ }^{3}$ Hackensack University Medical Center, Hackensack, NJ, USA. ${ }^{4}$ Stanford, CA, USA. ${ }^{5}$ Seattle Children's Hospital, Seattle, WA, USA.

${ }^{6}$ Boston, MA, USA. ${ }^{7}$ University of Alabama-Birmingham, Birmingham, AL, USA.

Published: 13 July 2012

\section{doi:10.1186/1546-0096-10-S1-A46}

Cite this article as: Ringold et al.: Disease characteristics and medication use in a multicenter cohort of children with juvenile idiopathic arthritis

(JA): preliminary analyses from the CARRAnet registry. Pediatric

Rheumatology 2012 10(Suppl 1):A46.

\section{Table 2}

\begin{tabular}{lll}
\hline Characteristic & $\begin{array}{l}\text { Mean } \\
\text { (Median) }\end{array}$ & Range \\
\hline Age at enrollment (years) & $11(12)$ & $<1-22$ \\
\hline Age at symptom onset (years) & $7(5)$ & $<1-16$ \\
\hline Age at first rheumatology visit (years) & $7(7)$ & $<1-21$ \\
\hline Disease duration (years) & $5(4)$ & $0-18$ \\
\hline $\begin{array}{l}\text { Duration between sympton onset and first } \\
\text { rheumatology visit (years) }\end{array}$ & $1(<1)$ & $0-12$ \\
\hline Number of active joints & $2(0)$ & $0-38$
\end{tabular}

\section{Submit your next manuscript to BioMed Central and take full advantage of:}

- Convenient online submission

- Thorough peer review

- No space constraints or color figure charges

- Immediate publication on acceptance

- Inclusion in PubMed, CAS, Scopus and Google Scholar

- Research which is freely available for redistribution

Submit your manuscript at www.biomedcentral.com/submit
C Biomed Central 